\title{
Relación entre la implementación del aprendizaje cooperativo y el logro en el aprendizaje individual de estudiantes universitarios
}

\author{
Relationship between the implementation of cooperative learning \\ and achievement in individual learning of college students
}

\author{
Mts. Fátima Valdez
}

\section{RESUMEN}

El presente trabajo reporta la investigación realizada con el objetivo de analizar la relación entre la implementación del aprendizaje cooperativo y el logro del aprendizaje individual de estudiantes universitarios. Las técnicas empleadas para la recolección de los datos fueron la encuesta y la observación, aplicadas por medio de dos cuestionarios semiestructurados y una guía de observación de clases a estudiantes de Psicopedagogía de la Universidad Iberoamericana, en el periodo de agosto a diciembre de 2010.

Los resultados permiten comprobar que el logro de los aprendizajes de los estudiantes del grupo experimental, con quienes se implementó la estrategia "aprendizaje cooperativo", se incrementa, tanto cualitativa como cuantitativamente.

Esta afirmación se fundamenta en que se visualiza que los estudiantes del grupo experimental han desarrollado las habilidades tales como: mejora de la capacidad de resolver problemas, toma de iniciativas y maduración en las relaciones con otros, realización de actividades en grupo, resolución de conflictos de manera constructiva, adecuación de los objetivos e intereses de cada uno a los del resto del grupo. Además, respeto a las opiniones diferentes al propio, actitudes de implicación y de iniciativa, comportamiento de acuerdo a los valores y normas que rigen las relaciones entre personas. Esto confirma que el aprendizaje cooperativo promueve el logro no sólo de habilidades de tipo cognitivo, sino también habilidades sociales y afectivas.

Palabras clave: aprendizaje cooperativo, logro de aprendizaje, habilidades cognitivas, sociales y afectivas. 


\section{ABSTRACT}

This paper reports on research conducted to analyze the relationship between the implementation of cooperative learning and achievement in individual learning of college students. Techniques for the collection of data included surveys and observation applied in the form of two semi-structured questionnaires and a class observation guide for psychopedagogy students at Universidad Iberoamericana. The data was collected from August to December 2010.

Results showed that achievement in learning from students in the experimental group, where the strategy "cooperative learning" was used, increases both qualitatively and quantitatively.

This statement is based on the fact that students from the experimental group developed skills such as: improvements in problem solving, initiative, and maturity in their relationships to others, constructive conflict resolution, and the capacity to adjust goals and interests to each group member. In addition they also developed respect for the opinions of others, initiative, and appropriate behavior with others. This confirms that cooperative learning promotes improvements in cognitive abilities as well as social and emotional skills.

Keywords: cooperative learning, learning achievement, cognitive, social and affective skills. 


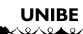

\section{INTRODUCCIÓN}

El proceso didáctico en cualquier nivel y más en el universitario es complejo y existen variadas exigencias a las que se debe dar respuesta, por tanto, es imperioso seleccionar una variedad de estrategias metodológicas, que garanticen la acción mediadora del profesor; quien se constituye en puente entre los contenidos del currículo institucional y las capacidades cognitivas de los alumnos, así mismo el papel que desempeñan los compañeros de aula, tanto en el proceso de socialización como en el proceso cognitivo de los estudiantes.

La presente investigación se considera actual, pues la labor educativa existente se dirige hacia la búsqueda de alternativas pedagógicas que se sustentan en el modelo socio-constructivo, siendo el aprendizaje cooperativo una herramienta esencial dentro de esas alternativas pedagógicas. El constructivismo representado por Piaget, Vygotsky, Novak y Ausubel confirma que el progreso en el desarrollo se efectúa gracias a la interacción social.

El aprendizaje cooperativo es una forma de trabajo en grupo basado en la construcción conjunta del conocimiento y el desarrollo de habilidades compuestas (aprendizaje y desarrollo personal y social), donde cada integrante del grupo es responsable no solo de su aprendizaje sino también del de los otros miembros del grupo. Además, las dinámicas internas que hacen que el aprendizaje cooperativo funcione, se basan en características que posibiliten a los docentes organizar las actividades de manera tal que los estudiantes se vuelvan positivamente interdependientes, individualmente responsables para hacer su trabajo, usen apropiadamente habilidades sociales y periódicamente procesen cómo pueden mejorar la efectividad de sus esfuerzos; por esa razón se ha tomado esta estrategia para el estudio y al respecto surge la siguiente interrogante de investigación:

¿Cuál es la relación entre la implementación de la estrategia aprendizaje en grupo cooperativo y el logro en el aprendizaje individual de estudiantes universitarios?

Se proponen además algunas preguntas secundarias para centrar la perspectiva de la investigación:

¿Cuáles son las características que presenta la implementación de la estrategia del aprendizaje cooperativo como proceso de enseñanza por parte del docente y los estudiantes? 
¿Qué factores explican el logro del aprendizaje con la utilización de la estrategia: aprendizaje cooperativo?

¿De qué manera se expresa el logro del aprendizaje cualitativo individual de estudiantes, en relación con la aplicación del aprendizaje cooperativo?

¿Cuál es el logro en cuanto al rendimiento académico cuantitativo individual de estudiantes, en relación con la aplicación del aprendizaje cooperativo?

El objetivo general de esta investigación es analizar la relación entre la implementación del aprendizaje en grupo cooperativo y el logro en el aprendizaje individual de los estudiantes universitarios.

Específicamente se busca: caracterizar la implementación de la estrategia del aprendizaje cooperativo por parte del docente y los estudiantes, reconocer los factores que explican el logro del aprendizaje con la utilización de la estrategia: aprendizaje cooperativo, explorar el logro del aprendizaje cualitativo individual de estudiantes y su relación con la aplicación del aprendizaje cooperativo y examinar el logro del aprendizaje en cuanto al rendimiento académico cuantitativo individual de estudiantes, y su relación con la aplicación del aprendizaje cooperativo.

La hipótesis planteada en esta investigación es "La implementación de la estrategia: aprendizaje cooperativo, incide incrementando el logro en el aprendizaje individual de estudiantes universitarios".

Acorde con la comprobación de la hipótesis planteada y los resultados obtenidos, se afirma que el aprendizaje cooperativo favorece el desarrollo de los estudiantes, tanto cualitativa como cuantitativamente ayudándoles a construir relaciones positivas entre ellos, trabajar en equipo, impulsar la creatividad, mejorar sus habilidades comunicativas y de análisis e incrementar su rendimiento académico cuantitativo; por tanto, debe ser asumido por los agentes educativos del proceso enseñanza-aprendizaje, en todos los niveles educativos y mayormente en el universitario.

\section{MATERIALES Y MÉTODOS}

Este trabajo de investigación se compone de un enfoque mixto (cualicuantitativo) con un alcance correlacional y explicativo.

Es correlacional porque tiene como propósito conocer la relación que existe entre dos variables en un contexto en particular y medir el grado de asociación entre ellas. Es explicativa porque pretende establecer 
las causas del evento o suceso que se estudia. Es transeccional porque recopila datos en un momento único, periodo de agosto a diciembre de 2010.

El diseño es cuasi-experimental, pues si bien se manipulan deliberadamente la variable independiente para observar su efecto y relación con una o más variables dependientes, los sujetos no se asignan al azar, sino que dichos grupos ya están formados antes del experimento, son grupos intactos. (Hernández et al., 2008).

El diseño cuasi-experimental empleado se realizó con prepruebaposprueba y grupos intactos (uno de ellos de control), pues sirvió para verificar la equivalencia inicial de los grupos y darle validez interna.

La población está integrada por 80 alumnos y 4 docentes de las carreras de Psicopedagogía de la Universidad Iberoamericana.

En cuanto a la muestra, para seleccionarla se consideró el grupo intacto de alumnos de los $2^{\circ}$ cursos de la carrera de Psicopedagogía, previa consulta de aceptación para participar de la investigación. Es una muestra no probabilística, denominada también dirigida ya que se tienen en cuenta las características de la investigación y la elección de sujetos de estudio depende del investigador. (Hernández et al., 2008). (p.241).

Este tipo de muestreo se denomina también opinático o intencional y según (Vieytes, 2004): "Se parte del supuesto de que las unidades seleccionadas son las más características o las que pueden proporcionar mayor información sobre la población, para estudiar un problema en particular" (p.403).

Las técnicas de recolección de datos utilizadas fueron: las encuestas y la observación. Los instrumentos utilizados fueron cuestionarios que se estructuraron con preguntas abiertas y cerradas de aplicación personal y autoadministrado y las guías de observaciones. Además, se utilizaron el programa y el plan de clase, los test estandarizado y no estandarizado, para el diagnóstico inicial y la verificación del resultado de aprendizaje.

Se procedió a validar los instrumentos con otro grupo de estudiantes de Psicopedagogía de la misma universidad, y luego se introdujeron ajustes para su aplicación final.

El procedimiento para el análisis de datos, se realizó de la siguiente manera: los datos del estudio cualitativo se transcribieron en sus formatos originales y se describieron cualitativamente y los datos del 
estudio cuantitativo fueron analizados estadísticamente, mediante Excel, versión 2007, en paquetes tipo SPSS versión 11.5, se realizaron cruces de variables y se presentaron los resultados en forma de tablas y gráficos.

En cuanto a los aspectos éticos, la realización de esta investigación respetó las normas de procedimiento para llevarla a cabo, contando con el consentimiento informado de cada sujeto de estudio y las autoridades de la universidad. Se garantizarán el anonimato de las personas y la confidencialidad de los datos en el proceso de la investigación y en la publicación de los resultados.

\section{RESULTADOS Y DISCUSIÓN}

Los resultados analizados fueron obtenidos mediante las encuestas administradas a alumnos (dos) y la observación de clases efectuada a los grupos experimental y de control; para ello se tuvieron en cuenta las preguntas de investigación, los objetivos, la hipótesis y las variables definidas para este trabajo. Se representaron los resultados mediante gráficos estadísticos.

Con relación a la variable 1- La implementación de la estrategia: aprendizaje cooperativo y su dimensión 1.- Roles del profesor, se observó, entre otros aspectos, lo siguiente:

Los datos del grafico 1 muestran que el $80 \%$ de los estudiantes del grupo experimental dice que sí, los docentes cumplen su rol de intervenir en los grupos para mejorar las relaciones interpersonales de los estudiantes, mientras que solo el $50 \%$ dice que sí lo hace en el grupo de control. Este rol del docente es de fundamental relevancia ya que posibilita contribuir a que el grupo tome conciencia de su dinamismo, monitoree la calidad de su cooperación y reflexione sobre los posibles modos de mejorar, si es necesario, lo cual se da en un bajo porcentaje en el grupo de control.

El docente debe monitorear el proceso para cuidar el desarrollo de las habilidades sociales que requiere la interdependencia positiva para aprender en grupo. Según Johnson y Johnson (1999), "La interdependencia positiva (cooperación) da como resultado la interacción promotora, en la que las personas estimulan y facilitan los esfuerzos del otro por aprender" (p. 8). 
Gráfico $\mathbf{N}^{\mathbf{0}} \mathbf{1}$

\section{Interviene en los grupos para mejorar} las relaciones interpersonales grupales

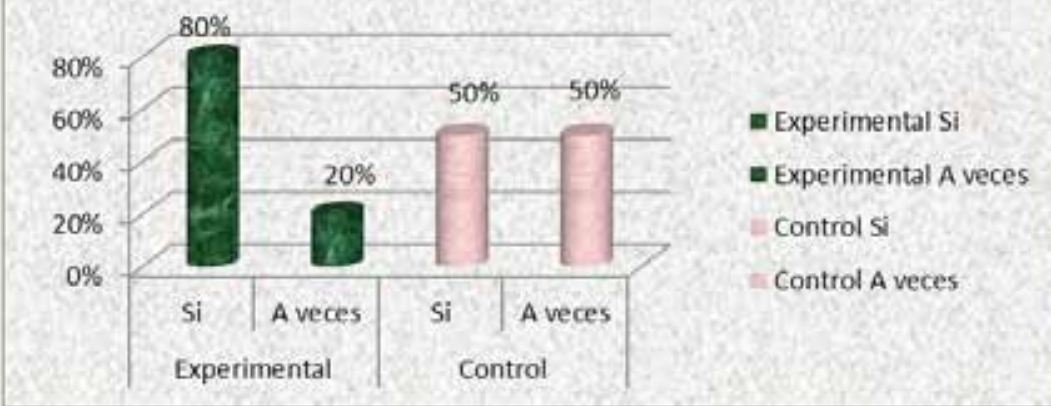

Fuente: Cuestionario aplicado a los estudiantes de Psicopedagogía

Es importante destacar que la correlación de Pearson indica que existe una fuerte correlación en cuanto al rol del profesor, en el grupo experimental y en el de control, por tanto, se deduce que el rol del profesor constituye uno de los pilares fundamentales en la implementación de la estrategia del aprendizaje cooperativo. Lo cual se demuestra en el gráfico 2.

\section{Gráfico $\mathbf{N}^{\circ}$ 2: Correlación de Pearson}

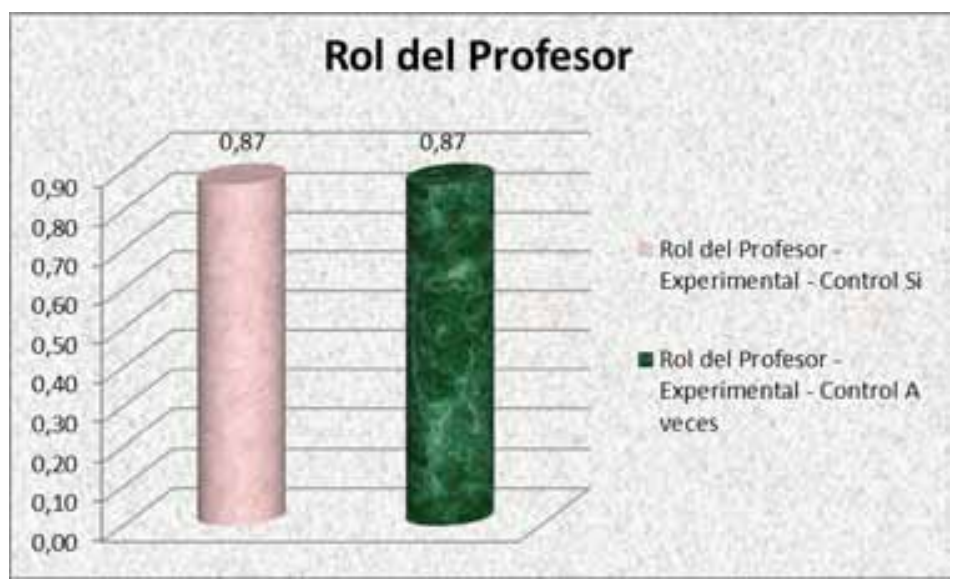


En cuanto a la dimensión 2- Actividades de los alumnos, de la variable 1, se destaca cuanto sigue:

Se observa en el Gráfico 3 que el $80 \%$ de los estudiantes del grupo experimental sí analizan y resuelven los problemas, mientras que solo el $40 \%$ de los estudiantes del grupo de control sí lo hace y el $60 \%$ lo hacen solo a veces. El análisis y la resolución de problemas constituyen capacidades logradas por los estudiantes cuando trabajan en grupo cooperativo, lo cual es fundamental para el aprendizaje de los mismos, hecho que se da en menor grado en los estudiantes del grupo de control.

En Woolfolk, A. (1996), se expresa que varios estudios nos demuestran que cuando la tarea implica un aprendizaje complejo y habilidades de solución de problemas, la cooperación lleva a mayor logro que la competencia, en especial en el caso de los estudiantes de menor capacidad (Johnson y Johnson, 1985; Slavin 1995).

\section{Gráfico $\mathbf{N}^{\mathbf{0}} 3$ :}

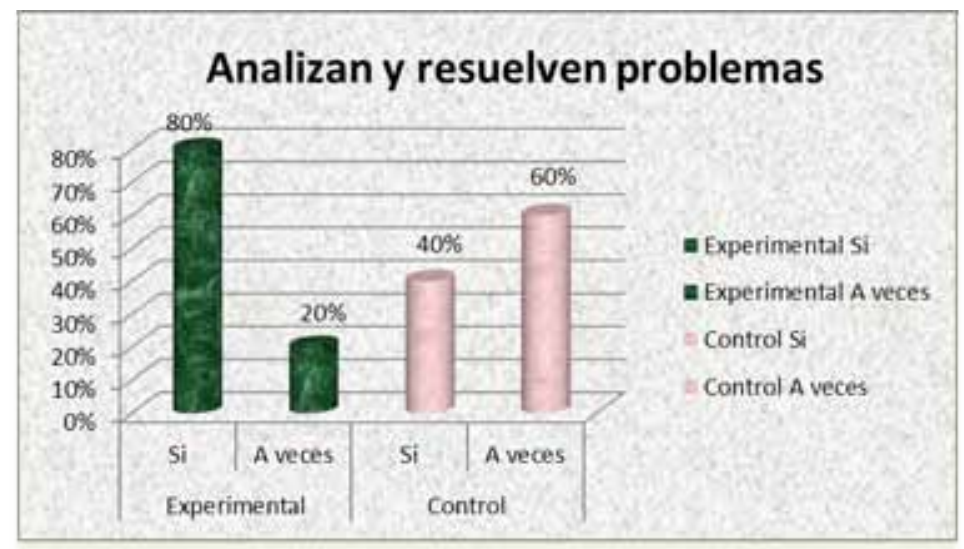

\section{Fuente: Cuestionario aplicado a los estudiantes de Psicopedagogía}

Se puede observar en el Gráfico 4 que si bien no existe una correlación positiva tan elevada, puede ser aceptada para los efectos de esta investigación, pues existen algunos aspectos de las actividades que se evalúan en la observación y que se considera dentro del rendimiento cualitativo, cuya correlación es elevada según se puede distinguir en el Gráfico 6. 


\section{Gráfico $N^{\circ} 4$ : Correlación de Pearson}

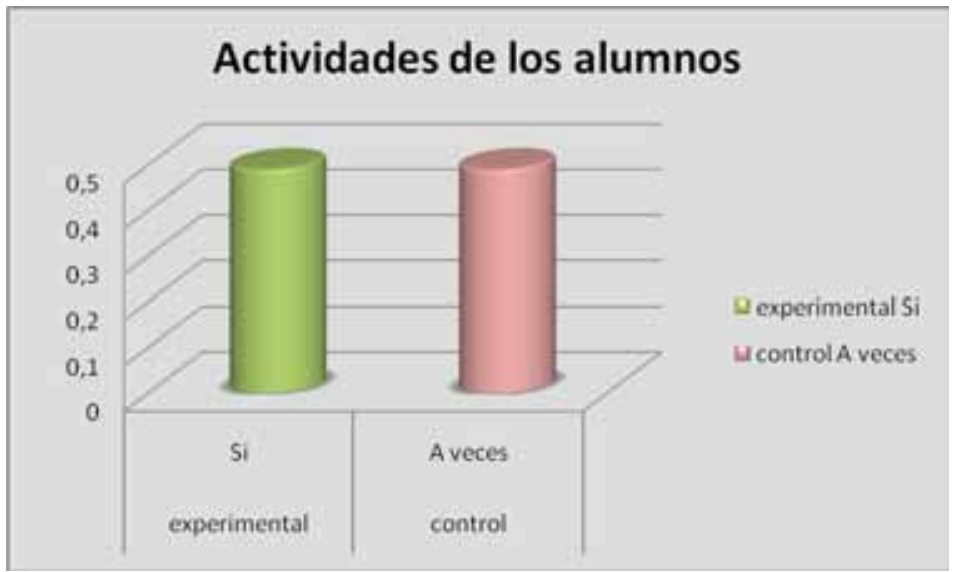

Referente a la variable 2. Logro en el aprendizaje de los estudiantes universitarios y su Dimensión 1. Rendimiento académico cualitativo, se observó, entre otros aspectos, lo siguiente: en el gráfico 5 el $80 \%$ de los estudiantes del grupo experimental afirman que demuestran actitud cooperativa, mientras que solo el $50 \%$ del grupo de control lo afirman, esto significa que los estudiantes del grupo de control están en desventaja frente a los del grupo experimental, en cuanto a la actitud cooperativa. Gros (2000) señala que "En un proceso de aprendizaje colaborativo, las partes se comprometen a aprender algo juntos. Lo que debe ser aprendido sólo puede conseguirse si el trabajo del grupo es realizado en colaboración".

Gráfico $\mathbf{N}^{\circ}$ 5:

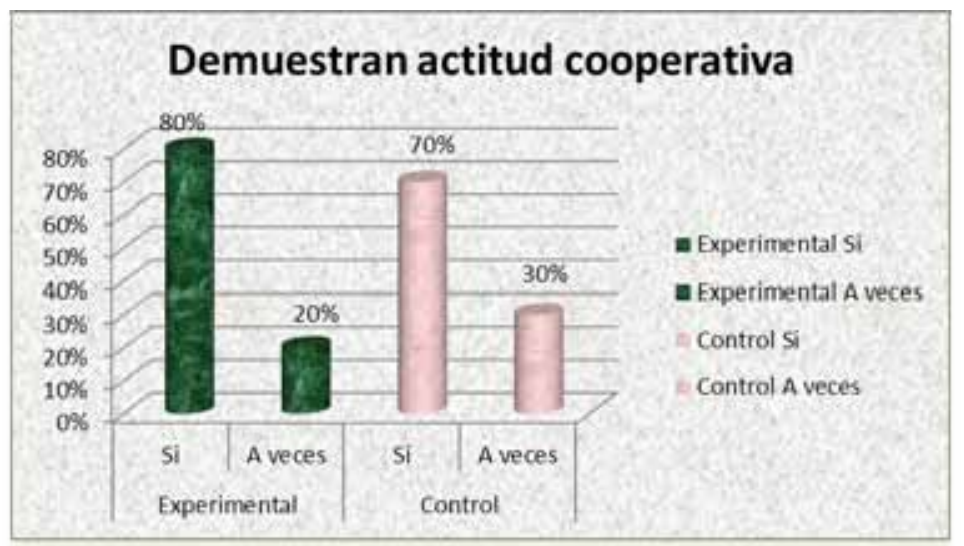

Fuente: Cuestionario aplicado a los estudiantes de Psicopedagogía. 
Según el Gráfico 6, el $100 \%$ de los estudiantes del grupo experimental observados, han logrado suficientemente el diálogo social, en cambio, solamente el $63 \%$ de los estudiantes del grupo de control. Esto se considera muy importante pues no solo se desarrolla la competencia cognitiva sino la social, lo cual se da en menor grado en los estudiantes del grupo de control. Al respecto, García, R., Traver, J. y Candela, I. (2001), señalan: "El trabajo cooperativo enseña estrategias y habilidades de cooperación en el aula y con los compañeros que facilitan la realización de aprendizajes por el propio alumnado aumentando su rendimiento escolar y fomentando actitudes de respeto tolerancia y colaboración" (pág. 4).

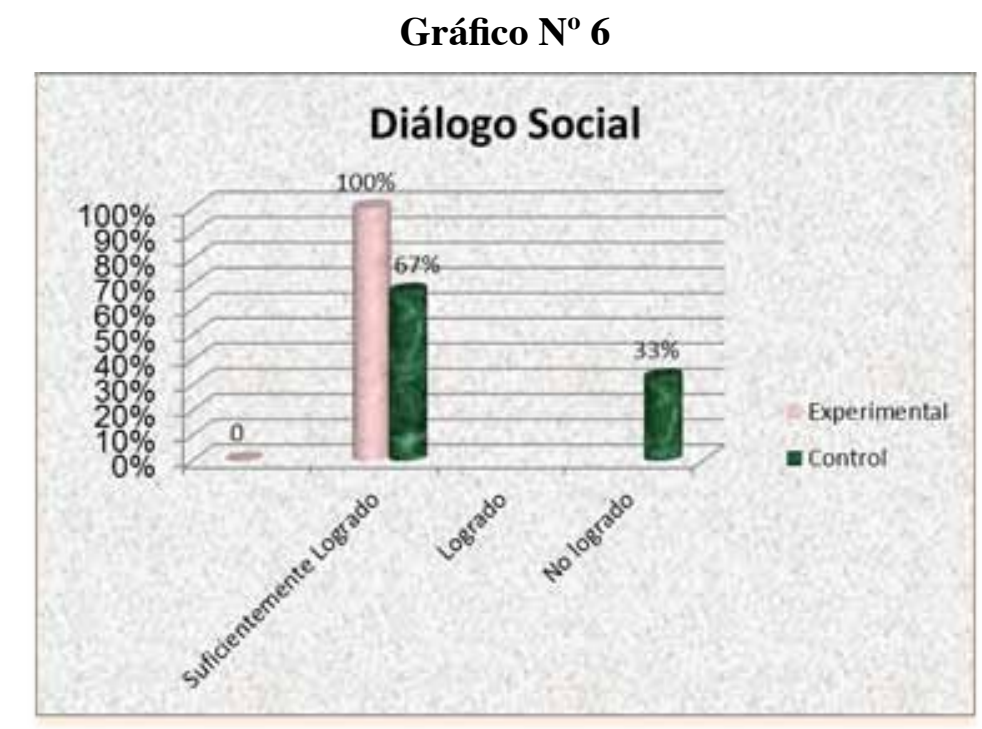

Fuente: Observaciones en el aula de clases a los estudiantes de Psicopedagogía

La correlación de Pearson, que se demuestra en el Gráfico 7, indica que existe una fuerte correlación en cuanto al rendimiento cualitativo de los grupos experimental y de control, lo cual confirma que los estudiantes con los que fue implementado el aprendizaje cooperativo tienen un grado de eficacia mayor en su rendimiento cualitativo. 


\section{Gráfico $\mathbf{N}^{\circ}$ 7: Correlación de Pearson}

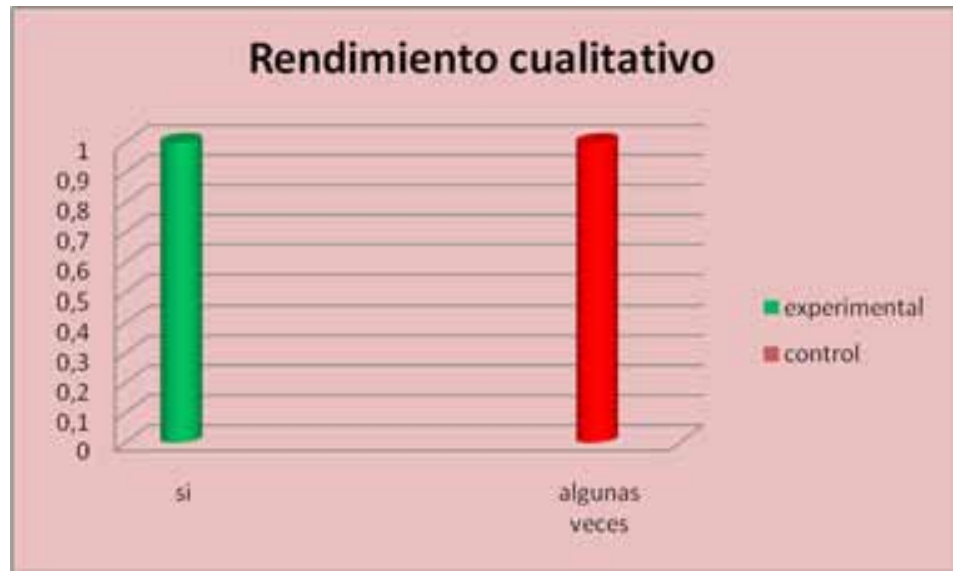

Además, se encontró que la correlación para el rendimiento cualitativo es alta, según el Gráfico 8, esto es fundamental porque se comprueba que los estudiantes del grupo experimental que implementaron el aprendizaje cooperativo tienen un rendimiento cualitativo elevado con relación al grupo de control.

\section{Gráfico $\mathbf{N}^{\circ}$ 8: Correlación de Pearson}

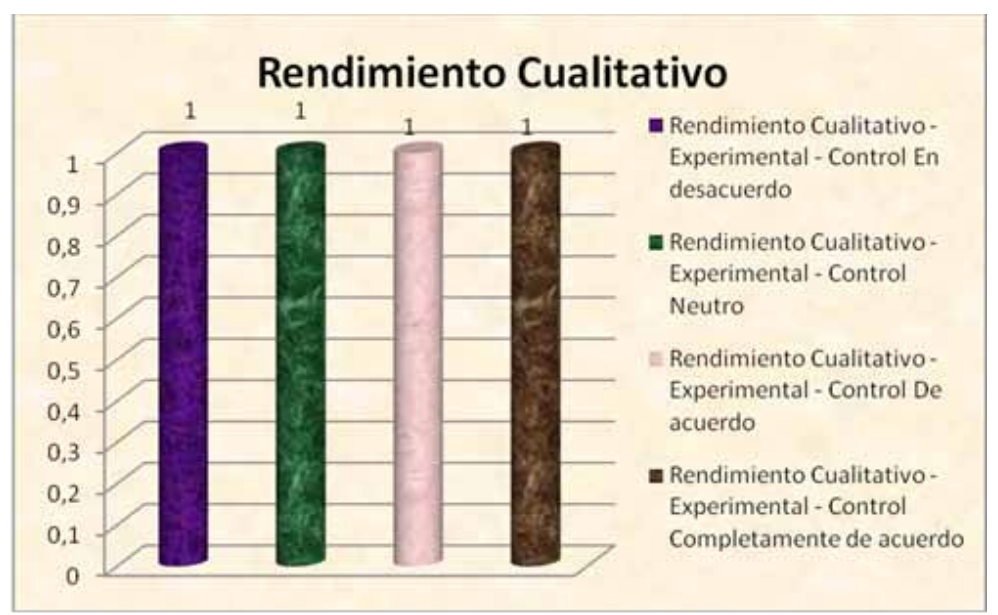


Con relación a la Variable 2. Logro en el aprendizaje de los estudiantes universitarios y su dimensión 2. Rendimiento académico cuantitativo, se verificó, según el Gráfico 9, que comparando las calificaciones hay una diferencia en cuanto al rendimiento del grupo cooperativo, si bien no muy alto pues en el grupo experimental hay un $70 \%$ que tiene calificación 5 (cinco), 30\% con calificación 4 (cuatro), mientras que en el grupo de control hay un $30 \%$ que tiene calificación 5 (cinco), $40 \%$ calificación 4 (cuatro) y 30\% calificación 3 (tres). Colomina (1990) dice que "el trabajo en equipo cooperativo tiene buenos efectos en el rendimiento académico de los participantes así como las relaciones socioafectivas que se establecen entre ellos"

\section{Gráfico No 9}

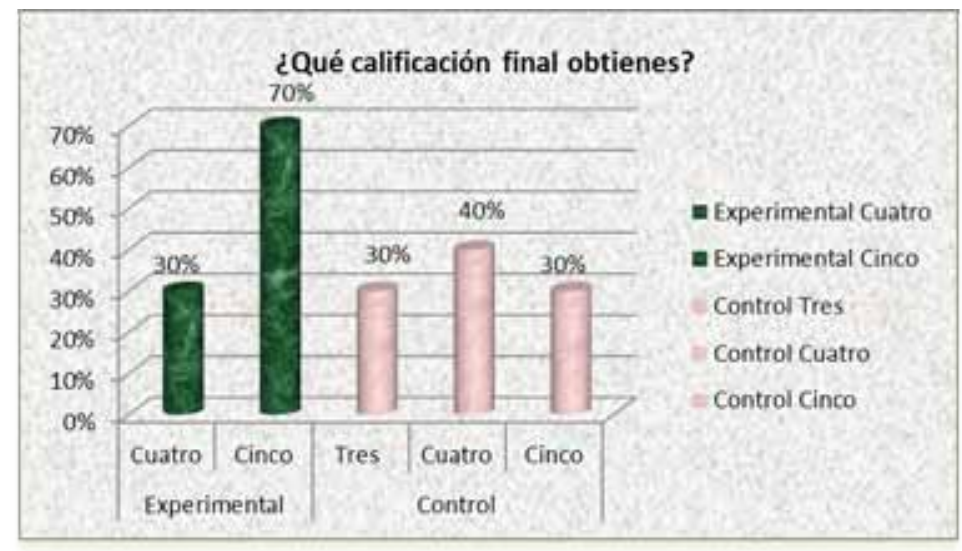

Fuente: Planilla de calificaciones de estudiantes de Psicopedagogía.

La correlación para el rendimiento cuantitativo del Gráfico 10 es alta, esto es fundamental porque corrobora que los estudiantes del grupo experimental que implementaron el aprendizaje cooperativo tienen un rendimiento cuantitativo más elevado con relación al grupo de control. 


\section{Gráfico N 10: Correlación de Pearson}

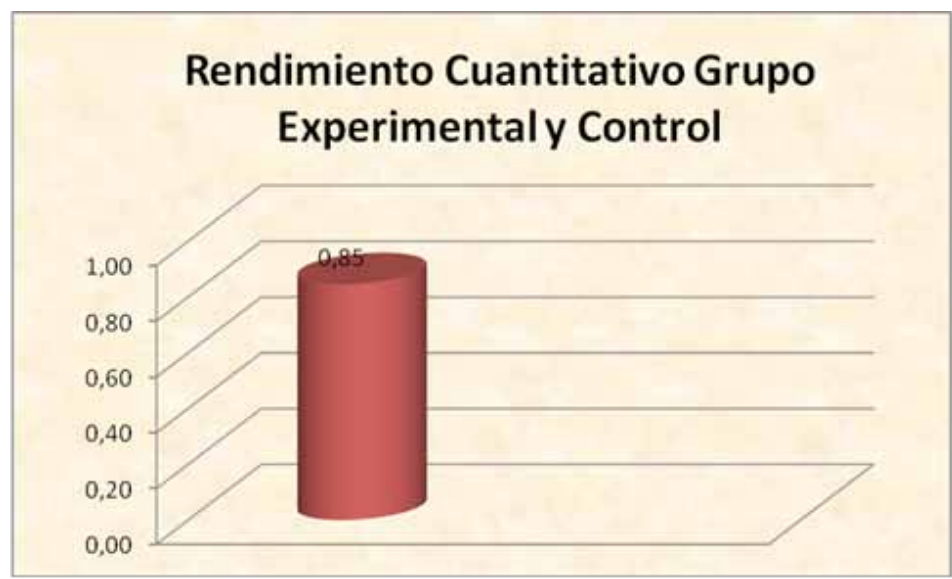

Según la prueba de Chi-Cuadrado Gráfico 11, se concluye con el valor de significancia que las variables del grupo experimental y control se ajustan a la hipótesis de que la implementación de la estrategia: aprendizaje en grupo cooperativo, incide incrementando el logro en el aprendizaje individual de estudiantes universitarios.

\section{Gráfico No 11}

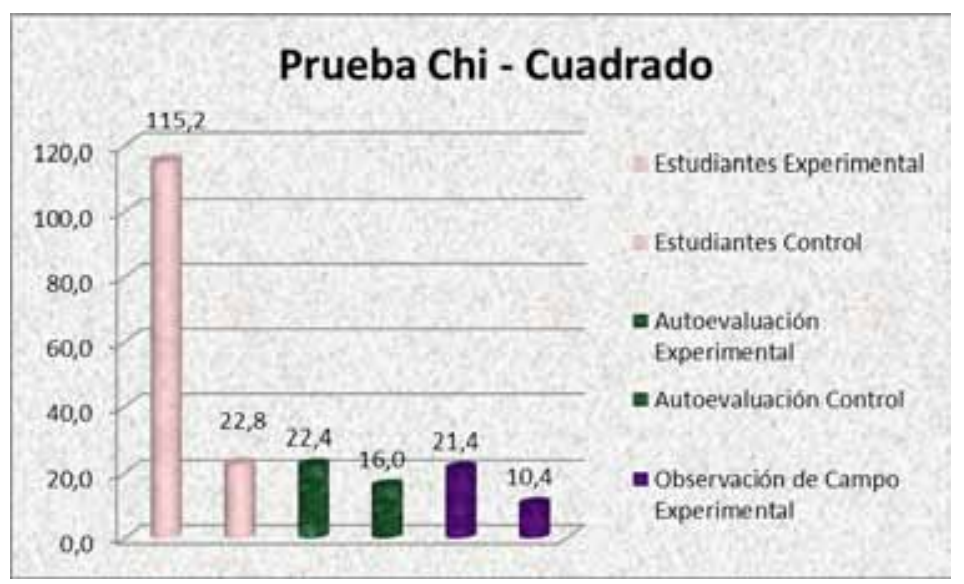




\section{CONCLUSIÓN}

A partir de los resultados obtenidos en la presente investigación, se puede afirmar que los objetivos planteados fueron logrados, ya que se pudo describir la relación entre la implementación del aprendizaje en grupo cooperativo, y el logro en el aprendizaje individual de estudiantes universitarios.

Los docentes cumplen varios roles en la implementación de la estrategia del aprendizaje cooperativo, según se desprende del análisis realizado el docente presenta el objetivo temático de cada actividad en forma verbal o escrita, explica la tarea y la interdependencia positiva a los alumnos, supervisa el aprendizaje e interviene en los grupos para apoyar la tarea, interviene en los grupos para mejorar las relaciones interpersonales grupales, recoge los documentos preparados por los estudiantes, evalúa y monitorea la actividad de los equipos. Es decir, pasa de administrador de la actividad a un observador empático que supervisa lo que hace cada equipo, brindando la ayuda necesaria en el momento oportuno y favoreciendo así la autorregulación de cada equipo; es decir, se convierte en un maestro mediador.

Esta concepción de maestro mediador se refuerza con la idea de Ferreiro (2004), quien afirma: En su rol el mediador el docente favorece el aprendizaje, estimula el desarrollo de potencialidades y corrige funciones cognitivas deficientes; es decir, en términos vygostkianos, mueve al sujeto que aprende en su zona de desarrollo potencial. Es decir, favorece el paso de un estado preliminar de no saber, poder o ser a otro cualitativamente superior de saber, hacer y ser.

Los estudiantes, por su parte, según los resultados del análisis realizado, comparten información y recursos, debaten y toman acuerdos, analizan y resuelven problemas, elaboran y entregan documentos tales como mapas conceptuales $\mathrm{u}$ otros recursos esquemáticos, comparten sus resultados con el resto de la clase, desarrollan el pensamiento crítico, y el diálogo social. Así, los estudiantes desempeñan un rol protagónico en las actividades, donde las tareas se solucionan en forma colectiva, en pequeños grupos, enfatizando la interacción educativa, el intercambio, y la confrontación de ideas, opiniones y experiencias entre estudiantes y docentes, durante el proceso didáctico.

Coll y Solé (1990) manifiestan: el concepto de interacción educativa como situaciones en donde los protagonistas actúan simultáneamente y 
recíprocamente en un contexto determinado, en torno a una tarea o un contenido de aprendizaje con el único fin de lograr objetivos claramente determinados.

Luego del análisis de los datos recogidos y la confrontación con el marco teórico, se deduce que la eficacia en cuanto a logros de aprendizaje con la aplicación de la estrategia: aprendizaje cooperativo depende de varios factores educativos, sociales y psicológicos.

Se pueden señalar algunas dimensiones que se consideran para explicar y comprobar la eficacia de estos factores mencionados al implementar las estrategias cooperativas, entre ellas están: La interdependencia positiva, la construcción de significado y las relaciones psicosociales.Estas consideraciones están teóricamente respaldadas por algunas concepciones como la de Johnson y Johnson (1999) quienes expresan: La interdependencia positiva (cooperación) da como resultado la interacción promotora, en la que las personas estimulan y facilitan los esfuerzos del otro por aprender.

Durante la aplicación del aprendizaje cooperativo, los estudiantes debaten los temas en clase, manifiestan capacidad para defender el tema propuesto en el grupo, desarrollan habilidades comunicativas, crítica constructiva, implicación, autonomía y creatividad, por tanto se considera que existe un grado elevado de eficacia del aprendizaje cualitativo individual de los mismos.

En el marco teórico se establece según Millis (1996), comparando los resultados de esta forma de trabajo, con modelos de aprendizaje tradicionales, se ha encontrado que los estudiantes aprenden más cuando utilizan el aprendizaje colaborativo, recuerdan por más tiempo el contenido, desarrollan habilidades de razonamiento superior y de pensamiento crítico y se sienten más confiados y aceptados por ellos mismos y por los demás.

Según los resultados del análisis, se concluye que los estudiantes del grupo experimental, con quienes se implementó la estrategia del aprendizaje cooperativo, presentan un nivel más elevado de rendimiento cuantitativo, con relación a los del grupo de control. Además, fue comprobado que no solo aumentó el rendimiento de tipo conceptual sino también el afectivo. Confrontando con el marco teórico, Colomina (1990) dice que el trabajo en equipo cooperativo tiene buenos efectos en el rendimiento académico de los participantes así como las relaciones socioafectivas que se establecen entre ellos. 
Se comprueba de este modo que el logro de aprendizajes en los estudiantes del grupo experimental se incrementa, tanto cualitativa como cuantitativamente. En mayor medida en forma cualitativa pues se visualiza que los estudiantes citados han desarrollado las habilidades de tipo social y afectivo, ya que se dio en ellos una mejora de la capacidad de resolver problemas, tomar iniciativas y madurar en las relaciones con otros, realizar actividades en grupo, adecuar los objetivos e intereses propios a los del resto del grupo, proponer normas y respetarlas, entender y respetar opiniones e intereses diferentes al propio, comportarse de acuerdo a los valores y normas que rigen las relaciones entre personas. Esto confirma que el aprendizaje cooperativo promueve no sólo el conocimiento de tipo conceptual, sino también habilidades de tipo social, afectivo y profesional.

La eficacia de implementación del aprendizaje cooperativo se explica mediante factores relacionados con la interacción directa de alumnosprofesor y alumnos entre sí que requiere por parte del docente el seguimiento de los procesos que van realizando los estudiantes, y una intervención diferenciada, en relación con lo que cada uno necesite. Además, una evaluación permanente de los trabajos realizados por los estudiantes que desarrollan la interdependencia positiva, la cual se produce mediante la realización de tareas comunes como por ejemplo, utilizar materiales de trabajo de manera compartida o la construcción de un producto grupal.

Los estudiantes desarrollan de esa manera su independencia tanto cognitiva como afectiva y a partir de ello despliegan habilidades sociales que los lleva a desarrollar una interdependencia social positiva posibilitándoles su crecimiento como personas.

Avalan estas premisas, diferentes teorías tales como la interdependencia social.de David W. y Roger T. Johnson, la teoría conductista del comportamiento humano, La teoría psicogenética de Jean Piaget, la teoría sociocultural de L. S. Vigotsky y la evolución de la concepción constructivista en la educación: El Constructivismo Social.

Además, se debe considerar que uno de los grandes aportes de la neurociencia a la educación es que explica la importancia de la participación de los estudiantes en el proceso de aprendizaje y de diversificar los modos de actividad y la comunicación en el salón de clase.

Según Ferreiro Gravié (2009), el aprendizaje cooperativo plantea una forma diferente de relacionarse maestro y alumnos en el proceso de aprender de este último. Ese modo de guiarlo es la mediación. 
Es necesario así mismo recordar que Barreto (1994) afirma que el aprendizaje cooperativo es aquel en que el alumno construye su propio conocimiento mediante un complejo proceso interactivo en el que intervienen tres elementos claves: los alumnos, el contenido y el profesor que actúa como facilitador y mediador entre ambos.

Por otra parte, Ferreiro (2009) refiere que: El aprendizaje cooperativo como forma de organización del proceso enseñanza-aprendizaje, propone una estructura de la lección con distintos momentos y estrategias de enseñanza que favorecen el trabajo con todo el cerebro, ya que intensifica y diversifica la participación de los alumnos en clase.

A partir de esta investigación se abren otras líneas de acción, por ejemplo, en cuanto al aprendizaje cooperativo y el uso de las TIC como herramientas tanto para las clases presenciales como en las virtuales a través de plataformas educativas.

\section{BIBLIOGRAFÍA}

AEBLI, H (200) 12 Formas básicas de enseñar. Una didáctica basada en la Psicología, Madrid, España, Narcea SA de Ediciones.

ANDER - Egg, E. (1997). El Trabajo en Equipo. Buenos Aires, Argentina, Editorial Lumen / Humanitas.

BARRETO, Violeta. (1994). "El aprendizaje: Enfoques y perspectivas". Bogota, Editorial Interamericana.

BOLAÑOS, G.; MOLINA BOGANTES, Z.. (2008) Introducción al currículo, San José de Costa Rica, EUNED.

BORSANI, María José, 2003, Adecuaciones curriculares: apuntes de atención a la diversidad, Edición $1^{a}$ ed. Publicación Buenos Aire, Novedades Educativas.

CARRETERO, Mario. (1993). "Desarrollo cognitivo y procesamiento de la información”, en Constructivismo y educación. Buenos Aires: Aique.

CARRETERO, Mario (2002), Construir y enseñar, Las ciencias experimentales, $3^{a}$ edición, Buenos Aires: Aique. 
CORONADO, M (2009), Competencias docentes. Ampliación, enriquecimiento y consolidación de la práctica profesional, Buenos Aires, Argentina, Noveduc.

Coll, C. y SOLE, I. (1990), La interacción profesor-alumno en el proceso de enseñanza-aprendizaje, Madrid, España, Alianza.

COLLC.; Colomina;ONARRUBIA,J.yROCHERA,M.,(1995)," Actividad conjunta y habla: una aproximación a los mecanismos de influencia educativa", en FERNÁNDEZ, P. y MELERO, M.A. (compiladores), La interacción social en contextos educativos. España. Siglo XXI.

DELORS, J. (1996), La educación encierra un tesoro, UNESCO. Los cuatro pilares de la educación. Editorial Santillana ISBN: 968-747400-9. Recuperado de http://ftp.ruv.itesm.mx/pub/sociales/competencias/ d5008_009.Pdf.

DE TORRE ZERMEÑO, Francisco (2005), 12 lecciones de pedagogía educación y didáctica; México, Alfa Omega editorial.

Diccionario de la Lengua Española - Real Academia Española (2001). $22^{\mathrm{a}}$ ed. Buenos Aires: Espasa Calpe, $2^{\mathrm{a}} \mathrm{v}$.

DÍAZ BARRIGA, F.; HERNÁNDEZ ROJAS, G. (2001), Estrategias docentes para el aprendizaje significativo, $2^{\mathrm{a}}$, Bogotá, Colombia Edición, Mac Graw Hill.

DÍAZ, F. (2006), Estrategias docentes para un aprendizaje significativo: una interpretación constructivista. México: McGraw Hill.

DRISCOLL, M.P. y VERGARA, A. (1997), Nuevas tecnologías y su impacto en la educación del futuro, en Pensamiento educativo, Santiago de Chile, Pontificia Universidad Católica.

Enerson, Diane M., R. Neill Johnson, Susannah Milner, and Kathryn M., Plank, (1997). "The Penn State Teacher II: Learning to Teach, Teaching to learn". University Park, PA, The Pennsylvania State University.

FERREIRO GRAVIÉ, R. (2009), Estrategias didácticas del aprendizaje cooperativo - Método Eli, $2^{\mathrm{a}}$ Edición, México: Trillas.

FERREIRO GRAVIÉ, R.; CALDERÓN, M. (2009), El abc del aprendizaje cooperativo, $2^{\mathrm{a}}$ Edición, México: Trillas.

FERREYRA, H. et all. (2006), Diseñar y gestionar una educación auténtica. México: Novedades Educativas. 
FLÓREZ, Rafael. (1997) Hacia una pedagogía del conocimiento. Santafé de Bogotá, Editorial McGrawHill.

GARCÍA, R., et, all (2001), Aprendizaje cooperativo. Fundamentos, características y técnicas, Madrid. Editorial CCS.

GROS, B. (2002), Constructivismo y diseños de entornos virtuales de aprendizaje. Revista de Educación.

HARGREAVES, D (1977), Las relaciones interpersonales en educación. Madrid, Ed. Narcea

HERNÁNDEZ, S. et al., (2008), Metodología de la investigación, cuarta edición, México, Mac Graw Hill.

HERNÁNDEZ, A, (1989) Metodología sistémica en la enseñanza Universitaria. Un proyecto de integración ecológica y pedagógica, Madrid, Ed. Narcea.

JOHNSON, D. (1999), El aprendizaje cooperativo en el aula, México. Ed. Paidós Educador.

JONES, F. et al., (1987) Estrategias para enseñar a aprender, $2^{a}$ Edición, Buenos Aires Argentina, ed. AIQUE.

LANDONE, E. (2002) Aprendizaje cooperativo en la clase de E/LE Recuperadode www.quadernsdigitals.net/datos web/hemeroteca/.../5899. html.

MARTÍN-KNIEP, G. (2001), Porfolios del desempeño de maestros, profesores y directivos. La sabiduría práctica. Buenos Aires: PAIDÓS.

MEDINA RIVILLA, A: SALVADOR MATA, F. (2004), Didáctica General, Madrid: Prentice Hall.

MILLIS, B. 1996. Materials presented at the university of Tennessee at Chattanooga instructional excellence retreat. Recuperado de http:www. utc.edu/Teaching-Resource_Center/cooplear.html.

NIETO GIL, J. (2004), Estrategias para mejorar la práctica docente, Madrid, CCS.

POSNER. G, (2004), Análisis de currículum, tercera edición, México: Mc Graw Hill.

ROMÁN PÉREZ, M. y DIEZ LÓPEZ (2.000), Aprendizaje y currículum. Diseños curriculares aplicados, Buenos Aires, Novedades Educativas, $6^{\text {a }}$ edición. 
ROMÁN PÉREZ, M. y otros. (2.001), Diseños curriculares de aula. Un modelo de planificación como aprendizaje - enseñanza, Buenos Aires, Novedades Educativas.

SALINAS, J. (2000). El aprendizaje colaborativo con los nuevos canales de comunicación Madrid, Síntesis.

SANTOS GUERRA, M. (1998) Evaluar es comprender, Buenos Aires, Magisterio del Río de la Plata.

SILBERMAN, M. (1998), Aprendizaje Activo, 101 estrategias para enseñar cualquier tema, Buenos Aires: Troquel.

VADILLO, G.; KLINGLER, C. (2004), Didáctica, teoría y práctica de éxito en Latinoamérica y España, México, Mc Graw Hill.

VIEYTES, R, (2004), Metodología de la Investigación en Organizaciones Mercado y Sociedad, Buenos Aires, Editorial de las Ciencias.

VILLAR ANGULO, L. (2005), Programa para la Mejora de la docencia universitaria, Madrid: Prentice Hall.

WOOLFOLK, A. (1995), Psicología educativa, $6^{\text {a }}$ Edición, Madrid: Prentice Hall. 